\title{
La teoría marxista de la dependencia desde una mirada feminista: un análisis sobre las leyes sobre aborto en el mundo
}

\author{
Agostina Costantino ${ }^{1}$ \\ Patricia Laterra ${ }^{2}$
}

Resumen: La teoría marxista de la dependencia surgió en la década del sesenta como una crítica tanto a las teorías neoclásicas del desarrollo, a las teorías del marxismo ortodoxo así como a las explicaciones estructuralistas sobre el desarrollo latinoamericano. A pesar de los importantes aportes que esta teoría realizó para pensar la posición de los países latinoamericanos en la acumulación global de capital, han sido escasas -sino nulas- las aplicaciones que la misma ha tenido en términos de pensar desde una perspectiva feminista esa misma acumulación. En este artículo, nuestro objetivo es realizar un aporte para pensar la función que cumplen las distintas legislaciones en torno al aborto en el mundo a la luz de la teoría marxista de la dependencia desde una perspectiva feminista. En términos generales, propondremos cuatro vías por medio de las cuales las condiciones actuales respecto a estos marcos legales garantizan diferentes formas de apropiación del valor por parte del capital en los distintos países del mundo.

Palabras clave: Teoría marxista de la dependencia; aborto; economía feminista.

Abstract: The marxist theory of dependency emerged in the 1960s as a critique of the neoclassical theories of development, theories of orthodox marxism as well as structuralist explanations of Latin

\footnotetext{
${ }^{1}$ Investigadora Asistente de CONICET en el Instituto de Altos Estudios Sociales (UNSAM). E-mail: agoscostantino@gmail.com.

${ }^{2}$ Lic. En Economía FCE-UBA. Docente de la cátedra optativa de Economía y Género en la FCE-UBA. E-mail: patricialaterra@gmail.com.
} 
American development. Despite the important contributions this theory made to think the position of the Latin American countries in the global accumulation of capital, there have been few applications that it has had in terms of thinking the feminist perspective in that accumulation. In this paper, our objective is to make a contribution to think about the role of different abortion laws in the world in the light of the marxist theory of dependency considering a feminist perspective. In general terms, we will propose four drivers in which the current conditions regarding these legal frameworks guarantee different forms of capital appropriation in the different countries of the world.

Keywords: Marxist theory of dependency; abortion; feminist economy.

\section{Introducción}

Si observamos la distribución mundial y las características de la legislación en torno al aborto, podemos identificar que en la mayoría de los países centrales la legislación es más permisiva, mientras que en los países dependientes estas leyes se tornan más restrictivas. Entendemos que las razones morales y simbólicas por las cuales no se permite el aborto en la mayoría de los países latinoamericanos y del Sur global en general no son suficientes para explicar esta distribución, siendo necesario incorporar elementos de Economía Política. Concretamente, creemos necesario indagar la reproducción material de la fuerza de trabajo, y sus diferencias en los distintos espacios de reproducción del capital.

El objetivo de este trabajo es proponer, de manera exploratoria, la hipótesis de que la legalidad del aborto y las políticas en derechos sexuales y reproductivos pueden entenderse, al menos parcialmente, por la funcionalidad que cumplen en la acumulación de capital a nivel mundial. No pretendemos demostrar una relación causal, sino una funcionalidad dentro de un sistema. En este sentido, a la luz de la teoría marxista de la dependencia (TMD), propondremos cuatro vías por medio de las cuales la disposición actual de los marcos

152 La teoría marxista de la dependencia... 
legales en torno al aborto a nivel mundial cumple las funciones de: (i) limitar el incremento del valor de la fuerza de trabajo en los países centrales; (ii) permitir el incremento de la sobrepoblación relativa o ejército industrial de reserva en los países dependientes; $y$ (iii) permitir la superexplotación del trabajo en los países dependientes.

La estructura del artículo es la siguiente: en la sección 2 describimos el panorama mundial de las leyes sobre aborto (su distribución y su grado de permisividad); en la sección 3 presentaremos el marco teórico que nos permitirá analizar este problema, la TMD; en la sección 4 presentaremos, a modo de hipótesis, las vías por medio de las cuales este marco legal cumple los fines económicos mencionados anteriormente; y por último, en la sección 5 presentamos algunas reflexiones finales.

Con miras a lograr un lenguaje inclusivo, en el presente trabajo nos referimos a las distintas identidades sexo-genéricas como "las/los" sabiendo y a nuestro pesar que, incluso, esta nomenclatura es binaria y no termina de incorporar las diferentes percepciones identitarias. El hecho de incorporar la dimensión de economía política en el análisis requiere para este trabajo particular el uso de estadísticas públicas, las cuales traen incorporado este sesgo binario que resulta difícil de eludir. Entendemos que es un desafío para la economía feminista poder trascender este sesgo en una herramienta que sigue siendo fundamental para analizar los comportamientos sociales.

\section{Panorama mundial de las leyes sobre aborto}

Si observamos la distribución mundial y las características de la legislación en torno al aborto puede notarse que la misma no es homogénea y que parece haber una diferencia muy clara entre los países. En particular, podemos identificar que en la mayoría de los países centrales la legislación es más permisiva, mientras que en los países dependientes y del sur global estas leyes se tornan más restrictivas.

153 La teoría marxista de la dependencia... 
Imagem 1: Mapa de la distribución de leyes de Aborto en el mundo año 2014

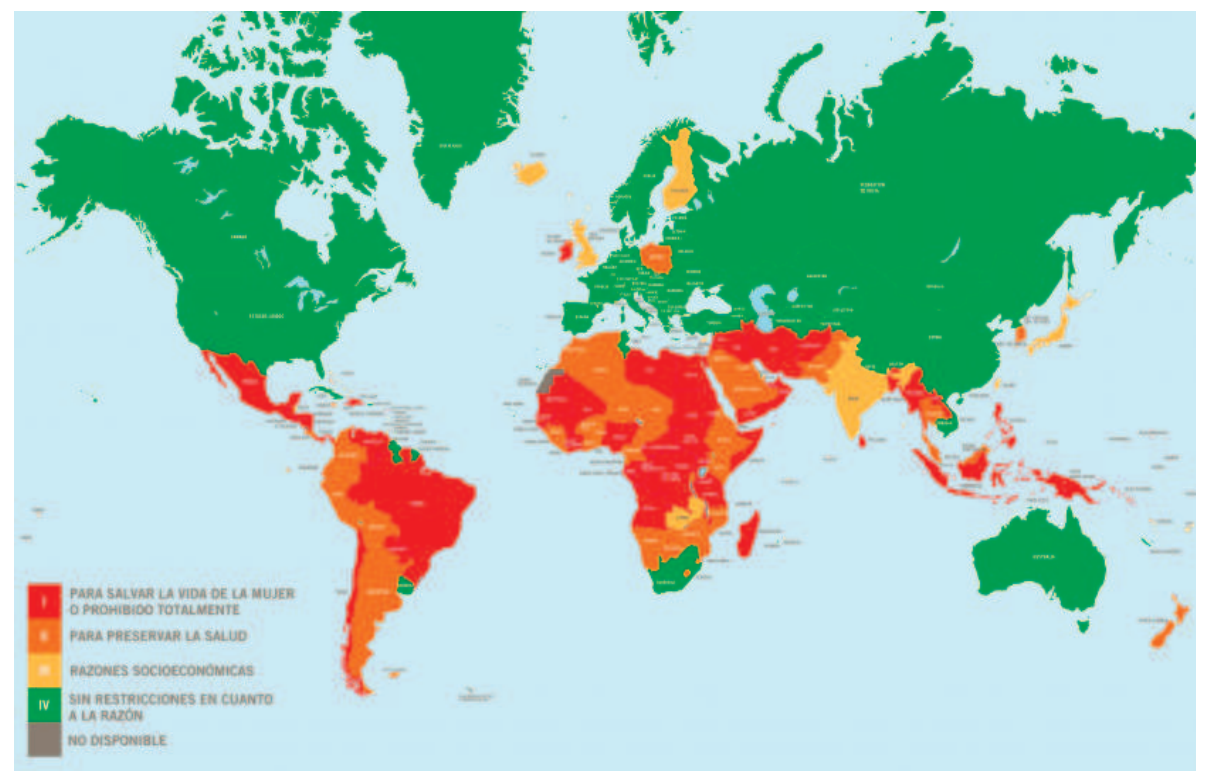

Fuente: Tomado de Centre for Reproductive Rights (2014).

El mapa confeccionado, para el año 2014, por el Centro de Derechos Reproductivos muestra la distribución de permiso o restricción total o parcial de leyes de aborto en el mundo. En rojo (I) se muestran los países que prohíben el aborto por completo o que sólo lo permiten para salvar la vida de la persona gestante; en naranja (II) se consigna a aquellos Estados que permiten el aborto para proteger la vida y la salud de la persona gestante; en amarillo (III) los países que permiten el aborto por razones socioeconómicas y en verde (IV) podemos observar los Estados con leyes más liberales donde está permitida la práctica de aborto sin restricción de razón (IV). Es necesario destacar que, las clasificaciones de los países no necesariamente indican el grado en que los servicios de aborto legal están realmente disponibles o permitidos en la práctica.

154 La teoría marxista de la dependencia... 
La mayoría de los países de la categoría IV, que permiten el aborto sin restricción en cuanto a la razón, son desarrollados y están ubicados en el Norte global, e incluyen Estados de Asia central y oriental (incluida China, el país más poblado del mundo). Son 61 países que comprenden el 39,5 por ciento de la población mundial. Varios países, como Estados Unidos, Canadá y Sudáfrica, reconocen explícitamente que una persona gestante tiene el derecho a elegir interrumpir su embarazo y que la interferencia excesiva con esta elección viola sus derechos constitucionales. Sin embargo, el Centro de Derechos Reproductivos (2014) alerta que es fundamental tener en cuenta que en la práctica, las leyes de aborto pueden aplicarse de manera diferente de lo que sugiere su categorización. En algunos países donde el aborto es permitido, la aplicación inadecuada de las disposiciones legales puede no garantizar su práctica de manera accesible y segura. Otra situación común es la interpretación de las leyes de aborto de manera más permisiva o restrictiva que la lectura literal que la ley sugiere. Es así como son centrales el apoyo público a los derechos al aborto, las opiniones de las y los funcionarios públicos, el personal efector de salud, entre otras circunstancias. Ejemplos de esto son los casos de Gran Bretaña e India que aún autorizando el aborto por razones socioeconómicas, en el primer caso se garantizan servicios de aborto libre y seguro y, en el segundo, la ley se ejecuta de forma inadecuada, ya que no se garantiza un acceso acorde a una práctica segura.

La mayor parte de los países latinoamericanos y africanos, casi todos los países musulmanes de Asia y cinco de los europeos (Islandia, Irlanda, Inglaterra, Polonia y Finlandia) pertenecen a las categorías I, II y III. Salvo los países europeos, por las razones que expondremos en el apartado siguiente, la mayoría puede considerarse dependientes. Es así como 66 países de la categoría I, que componen un $25,5 \%$ de la población mundial y que se encuentran principalmente en el Hemisferio Sur, con la excepción de varios países de Asia Central

155 La teoría marxista de la dependencia... 
y Oriental, prohíben por completo el aborto o lo permiten sólo para salvar la vida de la persona gestante.

Por otra parte, 59 países componen la categoría II que comprende al 13,8 por ciento de la población mundial y permiten el aborto para proteger la vida y la salud de la persona gestante. Las leyes de la mayoría de estos países autorizan el aborto por razones terapéuticas o de salud. Por último, la categoría III representa 13 países que permiten el aborto por razones socioeconómicas. Estos países se extienden por todo el mundo y abarcan aproximadamente el 21,3 por ciento de la población mundial. En general, las razones socioeconómicas para el aborto se consideran dentro del marco de la salud de las personas gestantes. Vale aclarar que como en el caso de lo descrito para la categoría IV el hecho que por determinadas razones sea legal la práctica eso no garantiza ni el acceso y ni condiciones seguras.

El análisis de la distribución de las leyes de acceso al aborto en el mundo no es concluyente para afirmar que hay una relación directa entre países dependientes y menor acceso al aborto por dos razones que detallamos anteriormente: por un lado, la existencia de leyes más permisivas no garantizan el efectivo acceso a la práctica y por otro el hecho de que no exista una plena legalidad o acceso a la práctica no impide que las personas con capacidad gestante aborten. Sin embargo, al incorporar al análisis la planificación (no)reproductiva y los embarazos no deseados podemos observar que las personas con capacidad gestante de países dependientes son las que más sufren las consecuencias de no poder gozar plenamente de los estándares de derechos sexuales y (no)reproductivos de los países desarrollados.

Entendemos que las razones morales y simbólicas por las cuales no se permite el aborto de manera irrestricta y la planificación (no) reproductiva que garantice la demanda de toda la ciudadanía en los países latinoamericanos y del Sur global en general no son suficientes para explicar esta distribución, siendo necesario incorporar elementos de Economía Política.

156 La teoría marxista de la dependencia... 
En el siguiente apartado, presentaremos algunos conceptos generales de la TMD para luego, en el apartado 4, presentar algunas de las funciones que, entendemos cumplen estos marcos legales en los distintos países.

\section{La TDM: ¿de qué se trata y para qué nos sirve?}

La TMD nace en la década del 60 como una crítica tanto a las teorías neoclásicas del desarrollo, a las teorías del marxismo ortodoxo así como a las explicaciones estructuralistas sobre el desarrollo latinoamericano.

La corriente de la dependencia afirmaba que la expansión del capitalismo a lo largo de la historia genera regiones, territorios y naciones diferenciadas desde el punto de vista de la apropiación y la generación del valor en países centrales (que son aquellos con mayor capacidad de apropiación del valor) y países dependientes o periféricos (que son aquellos que transfieren una gran parte del valor generado hacia los países centrales) (OSORIO, 2004b; GUNDER FRANK, 1979).

Ahora bien, ¿cómo funciona la condición de dependencia en la acumulación global del capital? En términos generales, la forma en la que se acumula capital en los países centrales es a través del aumento en la plusvalía relativa (OSORIO, 2014). Esto se logra por dos vías: aumentando la productividad en la producción de bienes-salario y aumentando las importaciones de estos mismos bienes producidos a un costo menor en otras economías. Estas dos vías permiten disminuir el salario requerido para cubrir las necesidades de las y los trabajadores en estos países (o sea, disminuir el valor de la fuerza de trabajo).

Los bienes producidos en estas economías, además, son consumidos por los propios asalariados. Por lo tanto, existe un interés por parte del capital en preservar el poder de compra de los mismos. En definitiva, en este tipo de economías la realización de la ganancia se hace a través de la venta a las propias personas trabajadoras.

157 La teoría marxista de la dependencia... 
Por su parte, en los países dependientes la realización de las ganancias se hace a través de la exportación de bienes-salario a los países centrales, o bien a través de la venta de bienes suntuarios a las clases altas locales (OSORIO, 2004a). Es decir, en este tipo de países, el capital se valoriza produciendo bienes que están fuera del alcance de las personas trabajadoras; por lo tanto, el capital puede atentar tranquilamente contra el poder de compra de éstas. Ruy Mauro Marini $(1979,1973)$ profundizó sobre el problema del intercambio desigual, sosteniendo que el deterioro en los términos de intercambio (producto de la existencia de monopolios en el sector industrial y los mayores aumentos de productividad en estas ramas) activaba "mecanismos de compensación" en los países dependientes para contrarrestar esa transferencia de valor hacia las economías centrales. Estos mecanismos, según el autor, consistían en aumentar la intensidad de explotación del trabajo (más producto en el mismo tiempo), aumentar la jornada laboral y pagarle a las y los trabajadores por debajo del valor de la fuerza de trabajo. ${ }^{3}$ A todos estos mecanismos, Marini les denominaba "superexplotación" del trabajo, y afirmaba que esto era una característica de los modos de producción en los países dependientes. Además, debido a la orientación externa de las estructuras productivas de estos países (que hace irrelevante el consumo de las y los trabajadores para la realización de las ganancias de los capitalistas), la tendencia es profundizar cada vez más el deterioro de las condiciones de los mercados de trabajo. Este deterioro debe entenderse siempre en términos relativos a las condiciones de valorización de la fuerza de trabajo a nivel mundial: no significa, necesariamente, un empeoramiento absoluto de las condiciones

\footnotetext{
${ }^{3}$ Con esto se quiebra el supuesto que utiliza Marx en El Capital acerca de que los salarios cubran la canasta de consumo necesaria para reproducir la fuerza de trabajo, supuesto con el cual buscaba demostrar la existencia de explotación incluso en presencia de intercambio de equivalentes. La presencia de un exceso de oferta de fuerza de trabajo en los países dependientes permite quebrar esta equivalencia, pagando al trabajador menos de lo que necesita para reproducir su capacidad de trabajar (OSORIO, 2012).
}

158 La teoría marxista de la dependencia... 
de vida, pero sí un rezago relativo al conjunto de bienes que compone la canasta de consumo de las y los trabajadores. Al mismo tiempo, la exportación de materias primas y alimentos baratos de los países dependientes a los países centrales permite también allí mantener bajas las remuneraciones laborales. ${ }^{4}$

En síntesis, una de las principales características de los países dependientes es que la acumulación de capital se logra de dos formas: (i) pagando salarios por debajo de los ingresos requeridos para cubrir las necesidades de supervivencia de las y los trabajadores; y (ii) extendiendo la jornada laboral, esto es aumentando la plusvalía absoluta (aunque pague un salario igual al valor de la fuerza de trabajo). Las dos medidas anteriores son posibles de aplicar en la medida en que exista una masa de trabajadores/as desempleados/as o subocupados/ as que permita un mercado de trabajo con estas características.

En el siguiente apartado analizaremos algunas funciones que, entendemos, cumple la forma en la que se distribuyen las distintas leyes sobre aborto y la planificación (no)reproductiva en el mundo (vista en el apartado 2) en la acumulación global de capital, siguiendo los conceptos principales vistos hasta aquí.

\section{La funcionalidad de la distribución de leyes sobre aborto en el mundo}

Luego de esta discusión teórica, presentaremos en este apartado una propuesta sobre las funciones que cumpliría la actual distribución de leyes sobre aborto en el mundo sobre la acumulación de capital a nivel global, y los canales por medio de los cuales estas funciones se llevan a cabo. Presentaremos cuatro canales o vías a modo

\footnotetext{
${ }^{4}$ Nótese la diferencia de las condiciones de trabajo que marca Marini: en los países dependientes se pagan salarios bajos porque estos salarios no permiten cubrir el valor de la fuerza de trabajo (es decir, el valor de la canasta de consumo del trabajador y su familia) mientras que en los países centrales también se busca pagar salarios bajos pero reduciendo el valor de esa canasta.
}

159 La teoría marxista de la dependencia... 
de cuatro hipótesis, es decir, lo siguiente no debe entenderse como una comprobación empírica sino como una propuesta coherente con el planteo teórico de la TMD para entender la funcionalidad económica de estos marcos legales.

En este sentido, entendemos que el actual marco regulatorio sobre el aborto en los distintos países del mundo cumple las funciones de (no de modo total, sino que apoya a fortalecer, junto con otros mecanismos): (i) limitar el incremento del valor de la fuerza de trabajo en los países centrales; (ii) permitir el incremento de la sobrepoblación relativa o ejército industrial de reserva en los países dependientes; y (iii) permitir la superexplotación del trabajo en los países dependientes. ${ }^{5}$ Estas funciones se llevan a cabo a través de los siguientes canales o vías:

\subsection{La vía del costo de vida en los países centrales}

La legalidad y la mayor amplitud de derechos en torno al aborto en los países centrales ha colaborado, junto con otras razones (culturales, sociales, políticas) al mayor control de la natalidad en aquellos países. Si bien las tasas de natalidad se encuentran en constante descenso en todos los países del mundo, en los países centrales ha caído más que en el resto. Por ejemplo, entre 1960 y 2015 la tasa de natalidad en la Unión Europea cayó un 46,3\%, en Estados Unidos un $47,7 \%$ y en Japón un 54,3\%; mientras que la caída promedio mundial en ese mismo periodo fue del 39,9\% (World Development Indicators, WORLD BANK).

La decisión de tener menos hijos/as por familia en este tipo de países está sustentada, entre otros motivos deseados y políticos, por el elevado costo de parir y criar niños/as. En Estados Unidos,

\footnotetext{
${ }^{5}$ Para una lectura en clave dependentista del papel de los sujetos feminizados en el proyecto neodesarrollista llevado a cabo en Argentina entre 2002 y 2016, ver Féliz y Díaz Lozano (2017). Los autores afirman que este proyecto se llevó a cabo (entre otras cosas) gracias al saqueo de las riquezas naturales, la superexplotación del trabajo y nuevas modalidades de apropiación del trabajo "reproductivo" realizado en gran medida por las mujeres.
}

160 La teoría marxista de la dependencia... 
el costo de un parto natural puede alcanzar los U\$S 30.000 y el de una cesárea los U\$S 50.000 para aquellos/as trabajadores/as que no tengan ningún tipo de cobertura de salud (BBC, 2015). En el año 2011, el 62\% de las mujeres que, en aquel país, contaban con algún plan de salud, no tenían cobertura por maternidad (debido a que el costo de incluir este tipo de cobertura aumenta en, aproximadamente, un $49 \%$ el costo total del seguro) (HERNÁNDEZ, s/f). Además, no sólo está el costo del alumbramiento sino también el de la crianza. También en Estados Unidos, según cálculos para el 2015, el costo de criar un hijo o hija desde que nace hasta los 17 años es de U\$S 233.610 ó U\$S 14.000 al año para una pareja con un ingreso medio. De la misma forma, en los países de la OCDE, el costo de criar un niño o niña va de U\$S 7.600 a U\$S 22.000 por hijo/a por año (en el Reino Unido, por ejemplo, esta cifra es de U\$S 306.629, hasta los 21 años) (CEACCU, 2013). No se trata de que sólo se impongan las razones económicas al momento de decidir criar un hijo/a, pero claramente es un motivo para evaluar dicha decisión, sobre todo en sociedades donde está mucho más presente la planificación familiar y la procreación. Según entrevistas realizadas por la CNN, las mujeres tienen en cuenta esto al momento de decidir tener hijos/as (CNN, 2013).

La decisión de tener menos hijos/as (o directamente, de no tener ninguno/a) en estos países, posibilitada por la legalidad y reglamentación en torno al aborto (además de la educación sexual y un mayor ejercicio de los derechos (no)reproductivos, repercute, por lo tanto, en un menor costo de vida en términos de núcleos familiares: el costo de vida y reproducción de una familia de 3 componentes será necesariamente menor al de una familia de 5 componentes. En este sentido, se impedirá que aumente considerablemente el valor de la fuerza de trabajo (que incluye el costo de vida y reproducción de toda la familia según las canastas típicas de consumo en cada tiempo y lugar) en los países centrales y, con ello, disminuirá la presión sobre la extracción de plusvalía relativa por parte del capital en estos mismos países.

161 La teoría marxista de la dependencia... 


\subsection{La vía de las cadenas globales de cuidados}

Las altas tasas de desocupación en los países dependientes obligan a que muchas personas (sin importar el género) tengan que migrar hacia países más desarrollados para poder garantizar el acceso a un ingreso y, con ello, la reproducción de su propia vida y familia. La particularidad que tienen este tipo de migraciones son las características de la inserción ocupacional de las mujeres: suelen hacerlo en tareas de cuidado y domésticas muy precarias y mal remuneradas. Según Canales (2014), en el año 2012, del total de las mujeres migrantes latinas en Estados Unidos, el 43,9\% se ocupaba en tareas de servicio doméstico, cuidado de personas y otros servicios personales; ${ }^{6}$ mientras que las mujeres nativas se ocupaban principalmente en actividades profesionales y de administración.

Es decir, este tipo de inserción ocupacional de las migrantes constituye lo que se conoce como "cadenas globales de cuidado", esto es redes transnacionales que se establecen para sostener cotidianamente la vida y a través de las cuales las mujeres se transfieren cuidados con base en ejes de jerarquización social (PÉREZ OROZCO, 2014). La crisis de los cuidados en los países del centro (falta de co-participación de los varones en las tareas de cuidados, la escasez de servicios públicos, la falta de regulaciones laborales que contemplen la garantía de provisión y cumplimiento de estas tareas) se conjuga con la crisis de reproducción social en los países del sur (el desempleo, la precariedad laboral, los bajos salarios, etc.), lo que confluye en la contratación, por parte de las mujeres de clase media-alta de los países del centro, de mujeres migrantes pobres provenientes de países dependientes. Según indican los estudios empíricos revisados (CANALES, 2014; SÁNCHEZ y SERRA YOLDI, 2013; CONNOR y MASSEY, 2011), sin estas cadenas no sería posible la reproducción social en los países más desarrollados: mientras las mujeres nativas pueden insertarse plenamente en

${ }^{6}$ Los "servicios personales" incluyen: servicios de limpieza, peluquería, estética y similares.

\begin{tabular}{c|l}
\hline 162 & La teoría marxista de la dependencia...
\end{tabular} 
el mercado laboral, la contratación de las tareas de cuidados a mujeres migrantes permite la reproducción de las familias y de las personas.

Como dijimos, la situación laboral de las migrantes que se insertan en este tipo de ocupaciones resulta muy precaria e insuficiente. Según los datos de Canales (2014), en Estados Unidos, el 35\% de las y los trabajadores no tiene acceso a ningún tipo de prestación de salud. Ahora bien, si se considera sólo el universo de mujeres migrantes latinoamericanas, este porcentaje asciende al $58 \%$; y es mucho más alto aún si sólo se consideran a las mujeres migrantes latinoamericanas ocupadas en servicios personales (71\%) o servicio doméstico (77\%). De la misma manera, el salario medio de la economía estadounidense en 2012 era de U\$S 22,35 la hora; mientras que para las mujeres migrantes latinoamericanas este salario ascendía a U\$S 14,10; y mucho más bajo aún para el caso de las mujeres migrantes latinoamericanas ocupadas en servicios personales (U\$S 9,6) o servicio doméstico (U\$S 8,9).

Por lo tanto, las condiciones de precariedad en las que se insertan las mujeres migrantes en las cadenas globales de cuidados permiten que el costo de reproducción de la vida en los países más desarrollados sea más bajo, y con esto, contribuyen también a mantener bajo el valor de la fuerza de trabajo en aquellos países. Es decir, incluso si las familias que contratan estos servicios se insertan en muy buenas condiciones laborales (en términos de seguridad social, estabilidad y remuneraciones) no requerirán de salarios muy altos para poder pagar por las tareas de cuidados.

\subsection{La vía de la tasa de desempleo "necesaria" para la superexplotación}

\section{del trabajo en los países dependientes}

Con respecto a la desocupación, ésta también se apoya en una mayor tasa de natalidad que implica mayores tasas de desocupación en los países dependientes y la superexplotación del trabajo o el aumento en la jornada laboral. La desigualdad en el acceso a los

163 La teoría marxista de la dependencia... 
derechos sexuales y (no)reproductivos y la distribución de las leyes de aborto en el mundo, aún con todas las salvedades que ya fueron explicitadas, tienen su correlato en las tasas de natalidad promedio según clasificación del Centro de Derechos reproductivos. En el gráfico podemos observar ante todo que las tasas de natalidad fueron descendiendo sustancialmente en los últimos 50 años. La tasa promedio mundial descendió de 31,84\% en 1960 a 19,35\% en 2014. Asimismo se puede observar que los países con categorías I y II están muy por encima del promedio mundial y los que poseen leyes más permisivas (III y IV) con una distancia sustancial de ese mismo promedio.

Imagem 2

Tasa de natalidad promedio según clasificación del Centro de Derechos reproductivos

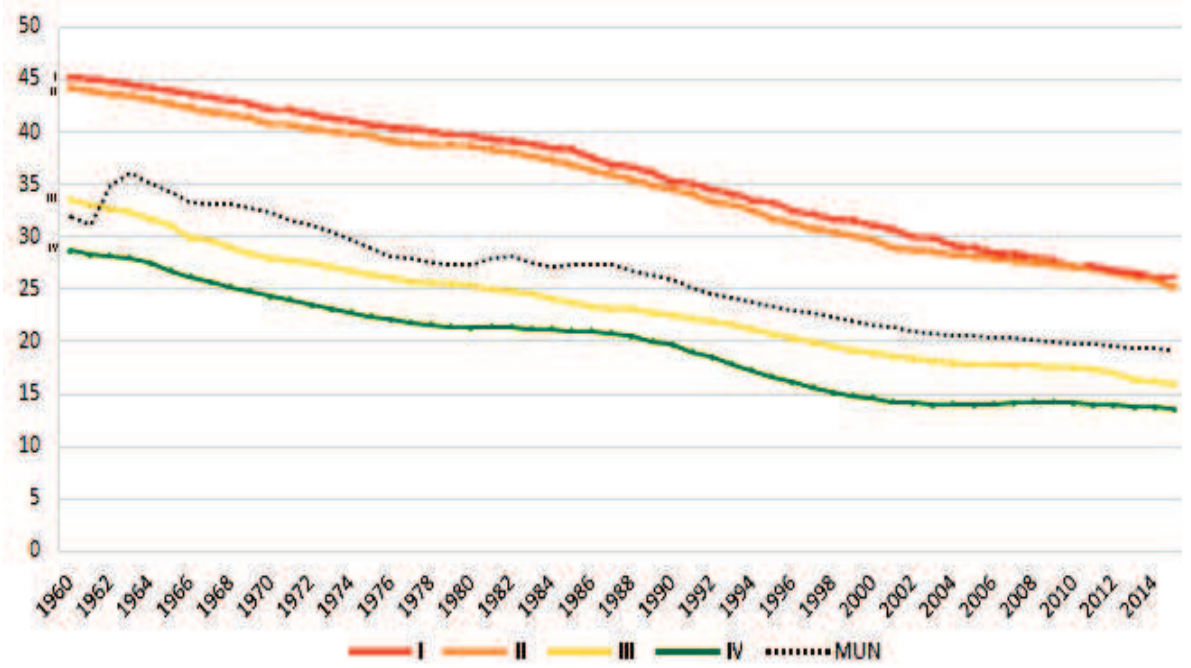

Fuente: Elaboración propia según base de datos del Banco Mundial y clasificación del Centro de Derechos Reproductivos para el Mapa de Aborto 2014.

164 La teoría marxista de la dependencia... 
Imagem 3

\section{Tasa de natalidad promedio según región}

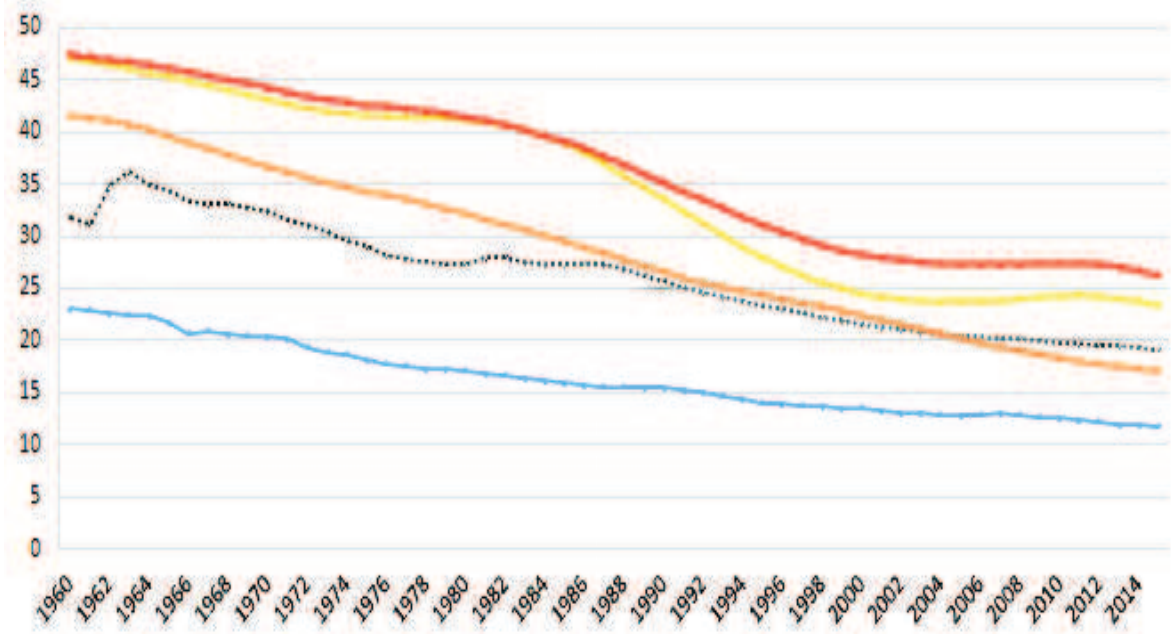

.........MUN $=$ OECD L Latinoamercay Caibe —-Medio Orientey Nortede África — Mundo Árabe

Fuente: Elaboración propia según base de datos del Banco Mundial y clasificación del Centro de Derechos Reproductivos para el Mapa de Aborto 2014.

Si analizamos las tasas de natalidad por región podemos observar que los países de la OCDE tienen tasas sustancialmente inferiores al promedio mundial. En contraposición, de las tres regiones que podemos caracterizar como dependientes que se muestran en el gráfico, sólo Latinoamérica se acerca, en los ' 90 , al promedio mundial pero muy por encima de las tasas de la OCDE. Con respecto a las tasas de desocupación, el análisis de casos puntuales de algunos países dependientes muestra tasas muy elevadas en comparación a países desarrollados. Estados Unidos, Reino Unido, Alemania y Suiza tienen trayectorias de desocupación muy por debajo al $10 \%$, siendo levemente superiores en épocas de crisis financieras como en el período 2008-2010. En contraposición, países dependientes como Sudáfrica, República

165 La teoría marxista de la dependencia... 
Dominicana, Puerto Rico y Colombia presentan tasas promedio de desocupación en el período estudiado de $24,1 \%, 15,6 \%, 13,7 \%$ y $11,4 \%$ respectivamente.

Imagem 4

Tasa de desocupación total

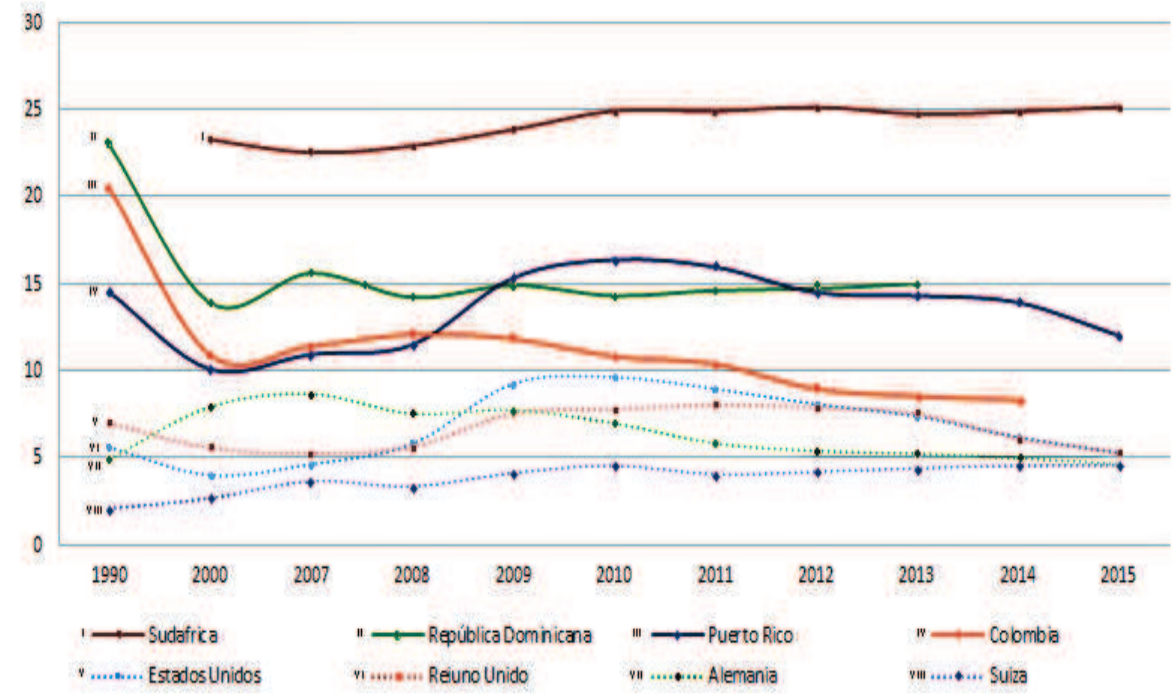

Fuente: Elaboración propia según base de datos del Banco Mundial y CEPALSTAT.

Claro está que estos mayores niveles de desocupación que, en promedio, existen en los países dependientes respecto a los centrales no son consecuencia únicamente de las mayores tasas de natalidad que existen también en los primeros respecto de los segundos. Otras razones, como las características de los eslabones industriales que son instalados en cada país, la intensidad del trabajo de las estructuras productivas, las posibilidades de inserción informal en el mercado de trabajo, entre otras, explican también este fenómeno. Sin embargo, la mayor tasa a la que se reproduce la población intensificará aún más los efectos provocados por las razones mencionadas anteriormente. Estos

166 La teoría marxista de la dependencia... 
niveles más altos de desocupación en los países dependientes respecto a los centrales, son una de las razones por las cuales en los primeros el capital puede ejercer la "superexplotación" del trabajo a la que aludía Marini.

Asimismo, una de las razones de las mayores tasas de natalidad en los países dependientes son los embarazos adolescentes o a edades tempranas. Esto incide profundamente en las trayectorias de vida de las jóvenes siendo las que más se les dificulta poder acceder al mercado de trabajo y a los empleos registrados, formales y con protección social. Esta dinámica ocurre tanto porque las trayectorias y la adquisición de credenciales educativas requisito declarado del mercado- se ven interrumpidas por la maternidad, como por la carga del trabajo doméstico y de cuidados no remunerado. El trabajo de cuidados no remunerado es un trabajo indispensable para que la fuerza de trabajo se pueda reproducir. Sin este trabajo no hay posibilidad de regeneración de la clase trabajadora (CARRASCO, 2006). Fundamentalmente en los países dependientes, este trabajo sigue recayendo injustamente en las espaldas de las mujeres, constituyendo un gran obstáculo para que las mismas puedan acceder a trabajos remunerados o insertarse pudiendo quebrar la segregación horizontal y vertical. Esto aumenta, también, las posibilidades de superexplotación del trabajo por parte del capital en la medida en que existe una mayor cantidad de trabajadoras "dispuestas" a vender su fuerza de trabajo en peores condiciones. Para los casos estudiados podemos observar que las tasas de desocupación femenina distan sustancialmente de las tasas de desocupación total en países dependientes. Para Sudáfrica, República Dominicana y Colombia las tasas promedio de desocupación en el período estudiado son del 26,97\%, 22,97\% y 14,64\% respectivamente, más altas que las tasas generales mencionadas anteriormente.

167 La teoría marxista de la dependencia... 


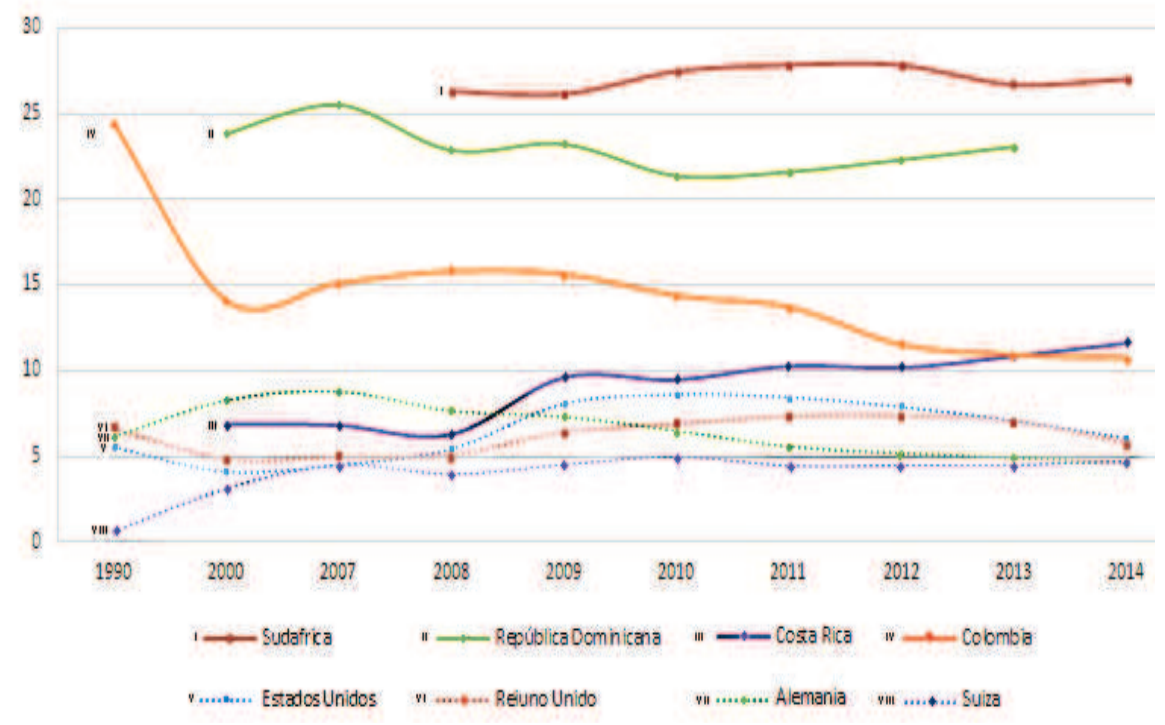

Fuente: Elaboración propia según base de datos del Banco Mundial y CEPALSTAT.

\subsection{Vía del costo de vida en los países dependientes}

Uno de los pilares para mantener bajo el valor de la fuerza de trabajo es o bien sostener la mayor cantidad de vidas a través del trabajo doméstico y de cuidados no remunerados o bien mantener bajo el salario de quien se dedica a tales tareas en el caso de que éste trabajo sea monetizado. En el caso de los países dependientes existe una fuerte incidencia en el tiempo de ocupación de las mujeres en trabajos domésticos y de cuidados no remunerados. Como fue analizado en el apartado anterior, una de las cuestiones fundamentales que explican tal dinámica es el peso del abandono en las trayectorias educativas y laborales de las mujeres (sobre todo, adolescentes) por embarazos tempranos, sin poder acceder a derechos sexuales y no reproductivos y a prácticas de aborto seguras y poco costosas. Esto garantiza que,

168 La teoría marxista de la dependencia... 
al insertarse de manera desigual en el mercado de trabajo, las tareas de cuidados sean menos monetizadas, abaratando los costos de vida y reproducción de los grupos familiares. Según ONU Mujeres (2015) y OIT (2017), para las economías desarrolladas el tiempo total que las personas empleadas, desempleadas o inactivas le dedican en el total del tiempo al trabajo doméstico y de cuidados no remunerados asciende en el caso de las mujeres a 4 horas y 20 minutos diarios frente a 2 horas y 16 minutos de los hombres. Para el caso de países con Encuestas de Uso del tiempo en Latinoamérica, ese número asciende a 5,56 horas total para las mujeres, estando el mayor peso relativo en las que poseen entre 6 y 9 años de instrucción educativa.

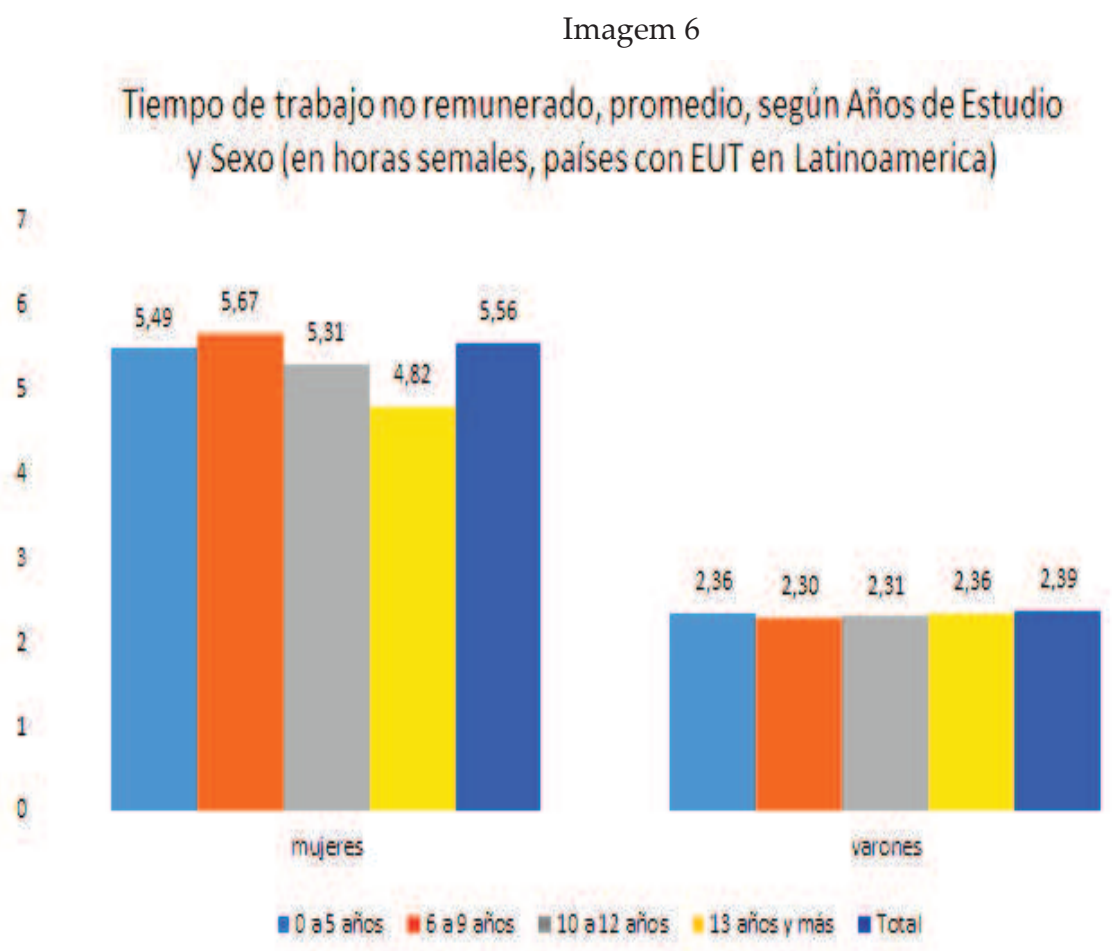

Fuente: Elaboración propia según base de datos CEPALSTAT.

169 La teoría marxista de la dependencia... 
Sin embargo, no desconocemos la necesidad del capital de tener que incorporar trabajadoras adicionales para poder sostener económicamente los grupos familiares. Según PNUD (2009) el ingreso laboral de las mujeres alivia la pobreza de manera evidente: en hogares donde las cónyuges no tienen ingresos propios, la incidencia relativa de la pobreza es mayor. Esto da por consecuencia dobles jornadas para las mujeres y la inserción en trabajos más precarizados y peor remunerados. El trabajo de las mujeres en el área productiva no ha alterado las responsabilidades en el área reproductiva, por lo que son las que más sufren cuando las dos esferas se encuentran y las más sensibles a la necesidad de una mejor integración de ambas (KABEER, 1998). Sin escapatoria, las mujeres se incorporan mayoritariamente al mercado de trabajo en sectores laborales feminizados o en trabajos de cuidados. En 2009, de cada cuatro mujeres en Latinoamérica tres trabajaban en el sector de los servicios y alrededor del $20 \%$ de la fuerza de trabajo femenina se concentraba en el servicio doméstico (ESPINO, 2012). Al mismo tiempo existen cada vez más hogares con presencia de familias con jefatura femenina. Alrededor de un tercio de los hogares de la región depende de los ingresos de una mujer que mantiene a su familia sin el apoyo de una pareja (PNUD, 2009).

\section{Reflexiones finales}

Este trabajo surge a raíz de pensar argumentos de apoyo en pos de una mayor igualdad en el mundo en torno al goce de los derechos sexuales y (no)reproductivos. Si bien este artículo es un primer paso para lograr nuestro propósito, somos cautas en advertir que los argumentos aquí esgrimidos no son concluyentes, pero dan apoyo a las razones simbólicas y culturales ya conocidas en torno a una mayor legalidad, acceso y despenalización del aborto en el mundo.

En términos generales, propusimos cuatro vías por medio de las cuales las condiciones actuales respecto a estos marcos legales garantizan diferentes formas de apropiación del valor por parte del

$170 \quad$ La teoría marxista de la dependencia... 
capital en los distintos países del mundo. Insistimos en que estas funciones no deben entenderse como causas de la existencia de estos marcos legales. Para ello, sigue siendo necesario tener en cuenta que la religiosidad y los patrones culturales continúan permeando la organización social, política, sexual y legal de la vida. No es casual que esta mayor religiosidad y menor autonomía sobre el cuerpo de las mujeres tengan una mayor incidencia en la regulación de la vida en los países dependientes.

Respecto del aporte teórico de nuestra contribución, se trata de una primera aproximación a pensar la TDM desde la perspectiva feminista. Al mismo tiempo, se trata de un aporte desde un punto de vista materialista a la economía feminista. Es necesario, sin embargo, seguir estudiando las vías por las cuales la diferente inserción en el mundo de los países tiene impactos en la organización social de la producción, el cuidado, la sexualidad y por ende en la sostenibilidad de la vida, una perspectiva que nos interesaría particularmente poder abordar con los entrecruces de ambas teorías. En específico creemos que hay líneas fundamentales para seguir pensando las cadenas globales de cuidados y TMD.

Uno de los desafíos planteados es aportar desde Latinoamérica un mayor desarrollo teórico en términos de economía feminista. La TMD, en este sentido, resulta un vehículo con gran valor para lograrlo, desde el momento en que dicha teoría se ha especializado en el aporte al conocimiento desde un punto de vista latinoamericano.

Por último, creemos que la economía feminista es una perspectiva fundamental para analizar y fortalecer la injusta desigualdad de géneros que tiene causas y correlatos a nivel material. La economía feminista es una disciplina relativamente joven dentro de las ciencias sociales y creemos que es necesaria la innovadora tarea de pensar y producir teoría. Es parte de nuestra responsabilidad como cientistas sociales hacer esfuerzos teóricos para poder complejizar los entramados problemas que vive nuestra sociedad.

171 La teoría marxista de la dependencia... 


\section{Referencias bibliográficas}

BBC. “¿Cuánto cuesta dar a luz en distintas partes del mundo?”. Febrero 20, 2015.

CANALES, A. "Migración femenina y reproducción social en los Estados Unidos. Inmigrantes latinas en los Estados Unidos". Revista Sociedad y Equidad, n. 6, 2014. http://revistas.uchile.cl/ index.php/RSE/article/viewArticle/27267.

CARRASCO, C. "La economía feminista: una apuesta por otra economía", disponible en http://www.americalatinagenera. org/es/index.php?option=com_docman\&amp;task $=$ doc_ download\&amp;gid=1004\&amp;Itemid=613. 2006

CEACCU. "Lo que cuesta tener un hijo". CEACCU, 2013.

CNN. "Criar un hijo en EEUU cuesta más de 240.000 dólares". CNN en español, agosto 15, sec. CNN Money, 2013.

CONNOR, P. e D. MASSEY. “La inserción en el mercado laboral de los inmigrantes latinos en España y en los Estados Unidos. Diferencias por país de origen y estatus legal". Revista Internacional de Sociología 69 (M1), p. 189-218, 2011. doi:10.3989/ ris.2011.iM1.391.

CENTRE FOR REPRODUCTIVE RIGHTS. "The World's Abortion Laws Map". Center for Reproductive Rights. Octubre 12, 2011. https://www.reproductiverights.org/document/the-worldsabortion-laws-map.

ESPINO, A. “Perspectivas teóricas sobre género, trabajo y situación del mercado laboral latinoamericano". En: V. ESQUIVEL (coord.) La

172 La teoría marxista de la dependencia... 
economía feminista desde América Latina: una hoja de ruta sobre los debates actuales en la región. GEMLAC - ONU MUJERES, 2012

FÉLIZ, M. e J. D. LOZANO. “Reproducción social, neodesarrollismo y saqueo de las riquezas sociales en Argentina, 2002-2016.". In: El neodesarrollo en debate. Crisis, transición y alternativas. Ensayo e investigación. El Colectivo, 2017.

GUNDER FRANK, A. Acumulación dependiente y subdesarrollo. México: Era, 1979.

HERNÁNDEZ, T. "Estados Unidos: Un país costoso para tener bebés". $\mathrm{s} / \mathrm{f}$.

KABEER, N. Realidades Trastocadas. Las jerarquías de género en el pensamiento del desarrollo. Buenos Aires: Editorial Paidós, 1998.

MARINI, R. M. Dialéctica de la dependencia. México: Era, 1973.

. "El ciclo del capital en la economía dependiente". En Mercado y dependencia, editado por Ursula Oswald. México: Nueva Imagen, p. 37-55, 1979.

OSORIO, J. Crítica de la economía vulgar. Reproducción del capital y dependencia. Colección América Latina y el Nuevo Orden Mundial. México: MA Porrúa, 2004a.

El Estado en el centro de la mundialización. La sociedad civil y el asunto del poder. México: Fondo de Cultura Económica, 2004b.

. Estado, biopoder, exclusión. Un análisis desde la lógica del capital. Barcelona y México: Anthropos-UAM Xochimilco, 2012.

173 La teoría marxista de la dependencia... 
"La noción de patrón de reproducción del capital". Cuadernos de Economía Crítica, n. 1, p. 17-36, 2014.

OIT. Las mujeres en el trabajo: Tendencias de 2016. Oficina Internacional del Trabajo - Ginebra: OIT, 2016.

OIT-PNUD. Trabajo y familia: Hacia nuevas formas de conciliación con corresponsabilidad social. Santiago, Oficina Internacional del Trabajo y Programa de las Naciones Unidas para el Desarrollo, 2009.

ONU Mujeres. El progreso de las mujeres en el mundo 2015-2016: transformar las economías para realizar los derechos. ONU Mujeres ed. Nueva York: ONU Mujeres, 2015.

PÉREZ OROZCO, A. Subversión feminista de la economía: aportes para un debate sobre el conflicto capital-vida. Madrid: Traficantes de Sueños, 2014.

SÁNCHEZ, M. J. e SERRA YOLDI, I. eds. Ellas se van: mujeres migrantes en Estados Unidos y España. Primera edición. México, D.F: Universidad Nacional Autónoma de México, Instituto de Investigaciones Sociales, 2013.

174 La teoría marxista de la dependencia... 